Chapman University

Chapman University Digital Commons

Mathematics, Physics, and Computer Science

Science and Technology Faculty Articles and

Faculty Articles and Research

Research

$1-17-2019$

Distribution Spaces and a New Construction of Stochastic Processes Associated with the Grassmann Algebra

Daniel Alpay

Ismael L. Paiva

Daniele C. Struppa

Follow this and additional works at: https://digitalcommons.chapman.edu/scs_articles

Part of the Algebra Commons, and the Other Mathematics Commons 


\section{Distribution Spaces and a New Construction of Stochastic Processes Associated with the Grassmann Algebra}

\section{Comments}

This article was originally published in Journal of Mathematical Physics, volume 60, issue 1, in 2019. DOI: $10.1063 / 1.5052010$

\section{Copyright}

AIP Publishing 


\section{Distribution spaces and a new construction of stochastic processes associated with the Grassmann algebra}

Cite as: J. Math. Phys. 60, 013508 (2019); https://doi.org/10.1063/1.5052010

Submitted: 13 August 2018 . Accepted: 26 December 2018 . Published Online: 17 January 2019

Daniel Alpay (D), Ismael L. Paiva (D), and Daniele C. Struppa

\section{ARTICLES YOU MAY BE INTERESTED IN}

Hermite polynomials and Fibonacci oscillators

Journal of Mathematical Physics 60, 012101 (2019); https://doi.org/10.1063/1.5040016

Notes on relative equilibria of isosceles molecules in classical approximation

Journal of Mathematical Physics 60, 012901 (2019); https://doi.org/10.1063/1.5028537

Non-self-adjoint operators with real spectra and extensions of quantum mechanics

Journal of Mathematical Physics 60, 012104 (2019); https://doi.org/10.1063/1.5048577

\section{Don't let your writing}

keep you from getting

published!

AIP Author Services 


\title{
Distribution spaces and a new construction of stochastic processes associated with the Grassmann algebra
}

\author{
Cite as: J. Math. Phys. 60, 013508 (2019); doi: 10.1063/1.5052010 \\ Submitted: 13 August 2018 - Accepted: 26 December 2018 • \\ Published Online: 17 January 2019
}



Daniel Alpay, ${ }^{1, a)}$ (D) Ismael L. Paiva, ${ }^{1, b)}$ (D) and Daniele C. Struppa ${ }^{2, c)}$

\begin{abstract}
AFFILIATIONS
'Schmid College of Science and Technology, Chapman University, One University Drive, Orange, California 92866, USA

${ }^{2}$ Donald Bren Distinguished Presidential Chair in Mathematics, Chapman University, One University Drive,

Orange, California 92866, USA

a) E-mail: alpay@chapman.edu

b) E-mail: depaiva@chapman.edu

${ }^{c}$ E-mail: struppa@chapman.edu
\end{abstract}

\section{ABSTRACT}

We associate with the Grassmann algebra a topological algebra of distributions, which allows the study of processes analogous to the corresponding free stochastic processes with stationary increments, as well as their derivatives.

Published under license by AIP Publishing. https://doi.org/10.1063/1.5052010

\section{INTRODUCTION}

In this work, we start the development in the Grassmann algebra of the counterpart of the noncommutative Fock space ${ }^{44}$ and of non-commutative stochastic distributions. ${ }^{5}$ Moreover, we study an analog of stochastic processes with stationary increments (such as the fractional Brownian motion) and their derivatives in the setting of the Grassmann algebra $\Lambda$. The processes introduced here differ from the ones discussed by Rogers in, e.g., Refs. 35-37.

We recall that $\Lambda$, also called the exterior algebra, is the algebra on a field $\mathcal{K}$ generated by a finite or countable set of elements $i_{n}$ not belonging to and linearly independent over $\mathcal{K}$ and satisfying

$$
i_{n} i_{m}+i_{m} i_{n}=0, \quad n, m=1,2, \ldots
$$

together with the identity element of $\mathcal{K}$. Usually, the field $\mathcal{K}$ is the setting of the complex numbers $\mathbb{C}$. We follow this choice.

Due to its role on supersymmetry, an element of $\Lambda$ is often referred to as a supernumber. When the number of generators is finite, say, $N$, we use the notation $\Lambda_{N}$ to evidence it. Note that for $M>N, \Lambda_{N}$ can be embedded in a natural way in $\Lambda_{M}$. In our approach, $\Lambda=\cup_{N \in \mathbb{N}} \Lambda_{N}$. This differs from the way $\Lambda$ is usually treated in the literature, where formal infinite sums are considered. Here, if $z \in \Lambda$, there exists $n(z)$ such that $z \in \Lambda_{n(z)}$. We will consider closures of $\Lambda$ to study cases with an effectively infinite number of generators. The construction of generators in the finite case follows with a matrix representation. In the infinite case, it is less straightforward, but concrete realizations can be given. ${ }^{12,34}$

We note that (1.1) also holds for many types of hypercomplex numbers if $n \neq m .{ }^{17}$ The difference here is that it also holds for $n=m$ so that

$$
i_{n}^{2}=0, \ldots \text { for } n=1, \ldots
$$


and, in particular, $\Lambda$ (and every $\Lambda_{\mathrm{N}}$ ) has divisors of 0 .

One can say that the analysis on this setting started in 1937 with Cartan showing that the Grassmann algebra can represent the exterior algebra if one introduces the idea of derivative (and multiplication) by its generators. ${ }^{15}$ In 1959, Martin considered supernumbers to study "classical versions" of physical functions for fermions and obtain their quantization through path integrals, ${ }^{30,31}$ an idea that would be later used by Schwinger to extend his quantum field theory to fermions. ${ }^{40}$ Moreover, in 1966, Berezin independently started an extensive study of what is now known as supermathematics. ${ }^{12,13}$

When working with $\Lambda_{N}$-and even with $\Lambda$ to a certain extent-only algebraic operations are involved, and there is no real problem of convergence since every element in $\Lambda$ generated only by $i_{n}$ 's is nilpotent. On the other hand, $\Lambda$ needs to be completed for various natural problems of analytical character.

The purpose of this work is to develop counterparts of classical notions from analysis and stochastic process theory when taking into consideration the completion of $\Lambda$ to a Hilbert space-denoted $\bar{\Lambda}^{(2)}$ (see Definition 3.4)-and embedding it in a Gel'fand triple

$$
\mathfrak{S}_{1} \subset \bar{\Lambda}^{(2)} \subset \mathfrak{S}_{-1} .
$$

The space $\mathfrak{S}_{-1}$, as we prove, has an algebra structure of the type that was first introduced by Kondratiev ${ }^{23}$ in the setting of Hida's white noise space theory and studied in a more generalized framework in Ref. 10. There are a number of parallels (and differences) between the present study and Refs. 6 and 7, where the complex numbers were replaced by the commutative algebra of Kondratiev stochastic distributions-see Ref. 23 for the latter and Refs. 21 and 22 for Hida's white noise space theory and the associated spaces of stochastic distributions. In those cases, the underlying space, i.e., the white noise space, is the commutative Fock space, which is typically associated with bosons. To obtain the so-called full Fock space, one includes the antisymmetric Fock space, which can be associated with fermions. In this context, i.e., when considering the full Fock space, it is also possible to define a noncommutative analog of the Kondratiev space. ${ }^{5,8}$ Moreover, the same type of tools can be developed in the framework of Q-deformed commutation relations. ${ }^{25}$ Here, we follow a similar approach, envisioning applications on stochastic processes and their derivatives.

As in Refs. 2 and 23 and more recently in the quaternionic setting, ${ }^{3}$ a Gel'fand triple together with its algebra structure allows one to consider functions from a compact metric space $E$, say, $[0,1]$, into $\bar{\Lambda}^{(2)}$. Such functions may be continuous, but not differentiable, when viewed as an element of $\bar{\Lambda}^{(2)}$. On the other hand, if $f$ is seen as a $\mathfrak{S}_{-1}$-valued function, one has, under a certain hypothesis, differentiability. The differentiability is, a priori, with respect to the strong topology of $\mathfrak{S}_{-1}$. However, it, in fact, happens in a Hilbert space, thanks to the assumed compactness of E. In particular, one can study stochastic processes and their derivatives in such spaces.

The main results of this article are as follows: First, in Sec. II, after discussing symmetries in $\Lambda$, we endow the latter with a family of norms and obtain new inequalities on these norms (see Theorem 2.16). Then, in Sec. III, we introduce the Fock space that can be associated with $\bar{\Lambda}^{(2)}$, making connections with classical aspects of superanalysis, e.g., left derivatives and Berezin integrals. Next, in Sec. IV, we embed the Fock space into Gel'fand triples given by (1.2) and prove that the product in $\mathfrak{S}_{-1}$ satisfies Våge-like inequalities (see Theorem 4.6). Finally, using those Gel'fand triples, we present in Sec. $V$ a close counterpart of the free stochastic processes with stationary increments and their derivatives.

\section{SYMMETRIES AND NORMS IN $\wedge$}

\section{A. Grassmann algebra and supernumbers}

Definition 2.1. We denote by $\mathfrak{I}$ the set of $t$-tuples $\left(a_{1}, \ldots, a_{t}\right) \in \mathbb{N}^{t}$, where $t$ runs through $\mathbb{N}$ and $a_{1}<a_{2}<\cdots<a_{t}$. For $\alpha=$ $\left(a_{1}, \ldots, a_{\mathrm{t}}\right) \in \mathfrak{I}$, we set $i_{\alpha}=i_{a_{1}} \cdots i_{a_{\mathrm{t}}}$ and write an element $z \in \Lambda$ as a finite sum

$$
z=z_{0}+\sum_{\alpha \in \mathfrak{I}} z_{\alpha} i_{\alpha}
$$

where the coefficients $z_{0}$ and $z_{a_{1}, \ldots, a_{\mathrm{t}}}$ are complex numbers.

The term that does not contain any Grassmann generator, $z_{0}$, is called the body of the number and is sometimes denoted by $z_{\mathrm{B}}$, while $z_{\mathrm{S}}=z-z_{\mathrm{B}}$ is said to be the soul of the number. ${ }^{16}$ One can also give a meaning to the sum (2.1) when it has an infinite number of terms, as we discuss in Sec. II C.

Sometimes it is convenient to define $i_{0}=1$ and "extend" the set $\mathfrak{I}$ to accommodate it. We will denote this new set $\mathfrak{I}_{0}$. Hence, a supernumber can be simply written as 


$$
z=\sum_{\alpha \in \mathfrak{I}_{0}} z_{\alpha} i_{\alpha}
$$

If $z=\sum_{\alpha \in \mathfrak{I}_{0}} z_{\alpha} i_{\alpha}$ and $w=\sum_{\beta \in \mathfrak{I}_{0}} w_{\beta} i_{\beta}$, their product makes sense since the sums are finite and can be written as

$$
z w=\sum_{\alpha, \beta \in \mathfrak{I}_{0}} z_{\alpha} w_{\beta} i_{\alpha} i_{\beta}
$$

Let $\alpha, \beta \in \mathfrak{I}$. Note that $i_{\alpha} i_{\beta}=0$ when $i_{\alpha}$ and $i_{\beta}$ have a common factor $i_{u}$, with $u \in \mathbb{N}$. Moreover, when $i_{\alpha} i_{\beta}$ does not vanish, it might still not be an element of the set $\left\{i_{\alpha}: \alpha \in \mathfrak{I}\right\}$, since permutations might be necessary to obtain such a type of element. However, because permutations only introduce powers of negative one, there exists a uniquely defined $\gamma \in \mathfrak{I}$ such that

$$
i_{\alpha} i_{\beta}=(-1)^{\sigma(\alpha, \beta)} i_{\gamma}
$$

where $\sigma(\alpha, \beta)$ is the number of permutations necessary to "build" $\gamma$ from $\alpha$ and $\beta$. If such a relation holds, we write

$$
\alpha \vee \beta=\gamma .
$$

So $i_{\alpha} i_{\beta}=(-1)^{\sigma(\alpha, \beta)} i_{\alpha \vee \beta}$. To rewrite it in a manner that includes the possibility of $i_{\alpha} i_{\beta}=0$, we define $\alpha \vee \beta=\emptyset$ if there is no $\gamma \in \mathfrak{I}_{0}$ such that (2.2) is satisfied. Then,

$$
i_{\alpha} i_{\beta}=(-1)^{\sigma(\alpha, \beta)} \sum_{\gamma \in \mathfrak{I}_{0}} \delta_{\alpha \vee \beta, \gamma} i_{\gamma}
$$

where $\delta_{\alpha \vee \beta, \gamma}$ is the Kronecker delta.

Remark 2.2. We note that $\mathfrak{I}_{0}$ defined as above is a monoid, with identity given by $\alpha=0$.

Remark 2.3. We note that $\mathfrak{I}_{0}$ is the counterpart of the set of indices $\ell$ considered in the case of infinitely many commuting (respectively, noncommuting) variables [see (3.2) and (3.3)].

It is important to observe that $\Lambda$ is a $\mathbb{Z}_{2}$-graded algebra. In fact, the elements that commute with each other are of the form

$$
z=z_{0}+\sum_{\substack{\alpha \in \mathfrak{I} \\|\alpha| \text { even }}} z_{\alpha} i_{\alpha},
$$

where $|\alpha|$ is the number of elements of $\alpha$. Those supernumbers are called the even supernumbers and their set is denoted by $\Lambda_{\text {even }}$. It is easy to verify that they commute with every element of $\Lambda$ and that, moreover, they form a commutative subalgebra.

On the other hand, the elements that anticommute with each other are of the type

$$
z=\sum_{\substack{\alpha \in \mathcal{I} \\|\alpha| \text { odd }}} z_{\alpha} i_{\alpha}
$$

They are known as odd supernumbers and do not form a subalgebra. In fact, it is an immediate result that the product of two odd supernumbers is an even supernumber. The set of odd supernumbers is denoted by $\Lambda_{\text {odd }}$.

The following results are easy, but they are relevant to our discussion.

Proposition 2.4. Let $v \in \Lambda_{\text {odd }} \subset \Lambda$. Then,

$$
v^{2}=0
$$

Proof. The proof follows easily. Let $v=\sum_{\substack{\alpha \in \mathfrak{I} \\|\alpha| \text { odd }}} v_{\alpha} i_{\alpha}$; 


$$
\begin{aligned}
v^{2} & =\frac{1}{2} \sum_{\substack{\alpha, \beta \in \mathfrak{I} \\
|\alpha|,|\beta| \text { odd }}}\left(v_{\alpha} v_{\beta} i_{\alpha} i_{\beta}+v_{\beta} v_{\alpha} i_{\beta} i_{\alpha}\right) \\
& =\frac{1}{2} \sum_{\substack{\alpha, \beta \in \mathfrak{I} \\
|\alpha|,|\beta| \text { odd }}} v_{\alpha} v_{\beta}\left(i_{\alpha} i_{\beta}+i_{\beta} i_{\alpha}\right) \\
& =0
\end{aligned}
$$

Remark 2.5. Even though Proposition 2.4 refers to the case where $v$ is an odd supernumber in $\Lambda$, its result is still valid when considering closures of $\Lambda$, i.e., when the set $\mathfrak{I}$ has an infinite number of elements.

Proposition 2.6. Let $N \in \mathbb{N}$ and consider $N+1$ elements $z_{n} \in \Lambda_{N}$ such that $z_{n B}=0$ for every $n \in 1, \ldots, N+1$. Then,

$$
\prod_{n=1}^{\mathrm{N}+1} z_{n}=0 .
$$

In particular,

$$
z_{\mathrm{S}}^{\mathrm{N}+1}=0
$$

for every $z=z_{\mathrm{B}}+z_{\mathrm{S}} \in \Lambda_{\mathrm{N}}$.

We note the following three corollaries, omitting the proof of the first two:

Corollary 2.7. Let $z \in \Lambda$ be such that $z_{B}=0$. Then, there exists $n=n(z)$ such that $z^{n(z)+1}=0$.

Corollary 2.8. The decomposition $z=z_{\mathrm{B}}+z_{\mathrm{S}}$ is the Jordan-Chevalley decomposition of $z$ for every $z \in \Lambda$.

Corollary 2.9. Let $z=z_{B}+z_{S} \in \Lambda$. Then, $z$ is invertible if and only if $z_{B} \neq 0$.

Proof. On the one hand, assume $z_{\mathrm{B}} \neq 0$. Then,

$$
z=z_{\mathrm{B}}\left(1+\frac{z_{\mathrm{S}}}{z_{\mathrm{B}}}\right)
$$

According to Corollary 2.7, there exists a $n(z)$ such that $z^{n(z)+1}=0$. Then,

$$
\left(1+z_{\mathrm{B}}^{-1} z_{\mathrm{S}}\right)^{-1}=\sum_{k=0}^{n(z)}\left(-z_{\mathrm{B}}^{-1} z_{\mathrm{S}}\right)^{k}
$$

and

$$
z^{-1}=z_{\mathrm{B}}^{-1} \sum_{k=0}^{n(z)}\left(-z_{\mathrm{B}}^{-1} z_{\mathrm{S}}\right)^{k} .
$$

On the other hand, assume $z$ is invertible and let its inverse be $w=w_{\mathrm{B}}+w_{\mathrm{S}} \in \Lambda$. Then, $z w=1$ and, in particular,

$$
z_{\mathrm{B}} w_{\mathrm{B}}=1 \Rightarrow z_{\mathrm{B}} \neq 0
$$

\section{B. Symmetries}

We can define a number of involutions of a supernumber. We start with the one characterized by

$$
\begin{aligned}
& i_{n}^{\dagger 1}=-i_{n}, \quad \forall n, \\
& (z w)^{\dagger 1}=w^{\dagger 1} z^{\dagger 1} .
\end{aligned}
$$


Therefore, the involution is defined as

$$
z^{\dagger 1}=z_{0}+\sum_{\alpha \in \mathfrak{I}} z_{\alpha} i_{\alpha}^{\dagger}
$$

Let

$$
\pi(\alpha)=\frac{|\alpha|(|\alpha|-1)}{2} .
$$

Then, $i_{\alpha}^{\dagger 1}=(-1)^{|\alpha|+\pi(\alpha)} i_{\alpha}$ and

$$
z^{\dagger 1}=z_{0}+\sum_{\alpha \in \mathfrak{I}}(-1)^{|\alpha|+\pi(\alpha)} z_{\alpha} i_{\alpha} .
$$

In general, we have

$$
\begin{gathered}
(z+w)^{\dagger 1}=z^{\dagger 1}+w^{\dagger 1}, \\
\left(z^{\dagger 1}\right)^{\dagger 1}=z .
\end{gathered}
$$

The next involution is given by the conjugation of the complex coefficients. The Grassmann generators are invariant under it, i.e.,

$$
i_{\alpha}^{\dagger 2}=i_{\alpha}
$$

Therefore,

$$
z^{\dagger 2}=\overline{z_{0}}+\sum_{\alpha \in \mathfrak{I}} \overline{z_{\alpha}} i_{\alpha},
$$

where the overline represents the usual conjugation of a complex number. In general, it holds

$$
\begin{aligned}
(z+w)^{\dagger_{2}} & =(z)^{\dagger_{2}}+(w)^{\dagger_{2}}, \\
\left(z^{\dagger 2}\right)^{\dagger_{2}} & =z, \\
(z w)^{\dagger^{2}} & =z^{\dagger 2} w^{\dagger_{2}} .
\end{aligned}
$$

The next involution is motivated by the fact already mentioned that $\Lambda$ is a $\mathbb{Z}_{2}$-graded algebra, i.e., the fact that an arbitrary supernumber $z \in \Lambda$ can be written as $z=u+v$, where $u \in \Lambda_{\text {even }}$ and $v \in \Lambda_{\text {odd }}$. We, then, define the involution $\dagger_{3}$ in a way that $u^{\dagger} 3=u$ and $v^{\dagger 3}=-v$. Hence, it holds in general

$$
z^{\dagger 3}=z_{0}+\sum_{\alpha \in \mathfrak{I}}(-1)^{|\alpha|} z_{\alpha} i_{\alpha}
$$

Observe that

$$
\begin{aligned}
(z+w)^{\dagger 3} & =(z)^{\dagger 3}+(w)^{\dagger_{3}}, \\
\left(z^{\dagger 3}\right)^{\dagger_{3}} & =z, \\
(z w)^{\dagger 3} & =(z)^{\dagger_{3}}(w)^{\dagger_{3}} .
\end{aligned}
$$

We note that the aforedefined involutions commute with each other

$$
\begin{aligned}
& \left(z^{\dagger_{1}}\right)^{\dagger_{2}}=\left(z^{\dagger_{2}}\right)^{\dagger_{1}}, \\
& \left(z^{\dagger_{2}}\right)^{\dagger_{3}}=\left(z^{\dagger_{3}}\right)^{\dagger_{2}}, \\
& \left(z^{\dagger_{3}}\right)^{\dagger_{1}}=\left(z^{\dagger_{1}}\right)^{\dagger_{3}} .
\end{aligned}
$$

Hence, four more involutions can be defined 


$$
\begin{aligned}
& z^{\dagger 4} \equiv\left(z^{\dagger 1}\right)^{\dagger 2}=\overline{z_{0}}+\sum_{\alpha \in \mathfrak{I}}(-1)^{|\alpha|+\pi(\alpha)} \overline{z_{\alpha}} i_{\alpha}, \\
& z^{\dagger 5} \equiv\left(z^{\dagger 2}\right)^{\dagger 3}=\overline{z_{0}}+\sum_{\alpha \in \mathfrak{I}}(-1)^{|\alpha|} \overline{z_{\alpha}} i_{\alpha}, \\
& z^{\dagger 6} \equiv\left(z^{\dagger 3}\right)^{\dagger_{1}}=z_{0}+\sum_{\alpha \in \mathfrak{I}}(-1)^{\pi(\alpha)} z_{\alpha} i_{\alpha}, \\
& z^{\dagger 7} \equiv\left(\left(z^{\dagger 1}\right)^{\dagger 2}\right)^{\dagger 3}=\overline{z_{0}}+\sum_{\alpha \in \mathfrak{I}}(-1)^{\pi(\alpha)} \overline{z_{\alpha}} i_{\alpha} .
\end{aligned}
$$

We call special attention to $\dagger_{7}$ because this is the involution that normally appears in the literature. ${ }^{16}$ Observe that it is very similar to $\dagger_{2}$ : it can be characterized as the complex conjugation of the coefficients and

$$
i_{n}^{\dagger 7}=i_{n}
$$

However, in general, $i_{\alpha}^{\dagger 7} \neq i_{\alpha}$. The reason for it is that given two supernumbers $z$ and $w$,

$$
(z w)^{\dagger 7}=(w)^{\dagger 7}(z)^{\dagger 7} .
$$

With such an involution, a real supernumber $z_{R}$ is defined as a supernumber with the property $\left(z_{R}\right)^{\dagger 7}=z_{R}$. Analogously, an imaginary supernumber $z_{C}$ is a supernumber such that $\left(z_{C}\right)^{\dagger 7}=-z_{C}$. With that, it is easy to see that a supernumber $z$ can be written as $z$ $=z_{R}+z_{C}$.

Remark 2.10. The involutions $\dagger_{k}, k=1,2, \ldots, 7$, together with the identity I form a commutative group with the composition law. In fact, it is the elementary Abelian group $E_{8}$.

Remark 2.11. It is clear that $z z^{\dagger_{k}}$ (and, in particular, the modulus $|z|_{k}^{2}=z z^{\dagger_{k}}$ induced by $\dagger_{i}$ ), with $k \in\{1,2, \ldots, 7\}$, is not a real number in general. To confirm it, first recall that $\uparrow_{4}, \uparrow_{5}, \uparrow_{6}$, and $\dagger_{7}$ are just compositions of $\dagger_{1}, \dagger_{2}$, and $\dagger_{3}$. Then, let $i \in \mathbb{C}$ be the complex unit and consider the supernumbers $z=i_{1} i_{2} i_{3}-i_{4}, w=i i_{1}+i_{2}$, and $r=1+i_{1} i_{2}+i_{3}$, which give

$$
z z^{\dagger 1}=2 i_{1} i_{2} i_{3} i_{4}, \quad w w^{\dagger 2}=2 i i_{1} i_{2}, \quad r r^{\dagger 3}=1+2 i_{1} i_{2} .
$$

Remark 2.12. The choices of $z$ and $w$ made in Remark 2.11 also show that

$$
z z^{\dagger 1} \neq z^{\dagger 1} z, \quad w w^{\dagger 2} \neq w^{\dagger 2} w
$$

Remark 2.13. In spite of Remark 2.12, $z z^{\dagger 3}=z^{\dagger 3} z$ for every $z \in \Lambda$. In fact, $z=u+v$ and, using Proposition 2.4,

$$
z z^{\dagger 3}=(u+v)(u-v)=u^{2}=(u-v)(u+v)=z^{\dagger 3} z .
$$

Remark 2.14. It is clear that the involutions defined here are continuous in $\Lambda_{\mathrm{N}}$ with respect to its natural topology induced by $\mathbb{C}^{2^{N}}$.

\section{Norms and completions}

Because the involutions defined above induce moduli that, in general, are not real, we introduce now the $p$-norm of a supernumber.

Definition 2.15. Let $p \geq 1$ be a real number. The $p$-norm of a supernumber $z \in \Lambda$ is defined as

$$
\|z\|_{p}=\left(\sum_{\alpha \in \mathfrak{I}_{0}}\left|z_{\alpha}\right|^{p}\right)^{1 / p},
$$

where $|\cdot|$ is the usual modulus of a complex number.

The above definition makes sense for any real $p \geq 1$. However, for the purposes of this paper, we consider only $p \in \mathbb{N}$. 
Theorem 2.16. Let $z, w \in \Lambda$. If $p=1$,

If $p>1$,

$$
\|z w\|_{1} \leq\|z\|_{1}\|w\|_{1}
$$

$$
\|z w\|_{p}^{p} \leq\|z\|_{1}^{p}\|w\|_{2^{p-1}} \prod_{k=1}^{p-1}\|w\|_{2^{k}}
$$

and

$$
\|z w\|_{p}^{p} \leq\|w\|_{1}^{p}\|z\|_{2^{p-1}} \prod_{k=1}^{p-1}\|z\|_{2^{k}} .
$$

Proof. The proof of (2.6) is straightforward and is, then, omitted.

To start the proof of (2.7), we note that

$$
\begin{aligned}
\|z w\|_{p}^{p} & =\left\|\sum_{\gamma \in \mathfrak{I}_{0}} \sum_{\alpha \vee \beta=\gamma}(-1)^{\sigma(\alpha, \beta)} z_{\alpha} w_{\beta} i_{\gamma}\right\|_{p}^{p} \\
& =\sum_{\gamma \in \mathfrak{I}_{0}}\left|\sum_{\alpha \vee \beta=\gamma}(-1)^{\sigma(\alpha, \beta)} z_{\alpha} w_{\beta}\right|^{p} \\
& \leq \sum_{\gamma \in \mathfrak{I}_{0}} \sum_{k=1}^{p} \sum_{\alpha_{k} \vee \beta_{k}=\gamma}\left|z_{\alpha_{1}}\right| \cdots\left|z_{\alpha_{p}}\right|\left|w_{\beta_{1}}\right| \cdots\left|w_{\beta_{p}}\right| \\
& \leq \sum_{\alpha_{1}, \ldots, \alpha_{p} \in \mathfrak{I}_{0}}\left|z_{\alpha_{1}}\right| \cdots\left|z_{\alpha_{p}}\right| \sum_{\substack { k=1 \\
\begin{subarray}{c}{\gamma \in \mathfrak{I}_{0} ; \exists \beta_{k} \in \mathfrak{I}_{0} \\
\alpha_{k} \vee \beta_{k}=\gamma{ k = 1 \\
\begin{subarray} { c } { \gamma \in \mathfrak { I } _ { 0 } ; \exists \beta _ { k } \in \mathfrak { I } _ { 0 } \\
\alpha _ { k } \vee \beta _ { k } = \gamma } }\end{subarray}}\left|w_{\beta_{1}}\right| \cdots\left|w_{\beta_{p}}\right| .
\end{aligned}
$$

Now, using the Cauchy-Schwarz inequality,



$$
\begin{aligned}
& \leq\left(\sum_{k=1}^{p-2} \sum_{\substack{\gamma \in \mathfrak{I}_{0} ; \exists \beta_{k} \in \mathfrak{I}_{0} \\
\alpha_{k} \vee \beta_{k}=\gamma}}\left(\left|w_{\beta_{1}}\right| \cdots\left|w_{\beta_{p-2}}\right|\right)^{4}\right)^{1 / 4} \times \\
& \times\left(\sum_{\substack{\gamma \in \mathfrak{I}_{0} \\
\alpha_{p-1} \vee \boldsymbol{\beta}_{p-1}}}\left|w_{\beta_{p-1}}\right|^{4}\right)^{1 / 4}\left(\sum_{\beta_{p} \in \mathfrak{I}_{0}}\left|w_{\beta_{p}}\right|^{2}\right)^{1 / 2} \\
& \leq\left(\sum_{\substack { k=1 \\
\begin{subarray}{c}{\gamma \in \mathfrak{I}_{0} ; \exists \beta_{k} \in \mathfrak{I}_{0} \\
\boldsymbol{\alpha}_{k} \vee \beta_{k}=\gamma{ k = 1 \\
\begin{subarray} { c } { \gamma \in \mathfrak { I } _ { 0 } ; \exists \beta _ { k } \in \mathfrak { I } _ { 0 } \\
\boldsymbol { \alpha } _ { k } \vee \beta _ { k } = \gamma } }\end{subarray}}\left(\left|w_{\beta_{1}}\right| \cdots\left|w_{\beta_{p-2}}\right|\right)^{4}\right)^{1 / 4}\left(\sum_{\beta_{p-1}}\left|w_{\beta_{p-1}}\right|^{4}\right)^{1 / 4}\|w\|_{2} \\
& \leq\left(\sum_{\substack { k=1 \\
\begin{subarray}{c}{\gamma \in \mathfrak{I}_{0} ; \exists \beta_{k} \in \mathfrak{I}_{0} \\
\boldsymbol{\alpha}_{k} \vee \boldsymbol{\beta}_{k}=\gamma{ k = 1 \\
\begin{subarray} { c } { \gamma \in \mathfrak { I } _ { 0 } ; \exists \beta _ { k } \in \mathfrak { I } _ { 0 } \\
\boldsymbol { \alpha } _ { k } \vee \boldsymbol { \beta } _ { k } = \gamma } }\end{subarray}}\left(\left|w_{\beta_{1}}\right| \cdots\left|w_{\beta_{p-n}}\right|\right)^{2^{n}}\right)^{1 / 2^{n}} \prod_{k=1}^{n}\|w\|_{2^{k}} \\
& \leq\left(\sum_{k=1}^{2} \sum_{\substack{\boldsymbol{\gamma} \in \mathfrak{I}_{0} ; \exists \beta_{k} \in \mathfrak{I}_{0} \\
\boldsymbol{\alpha}_{k} \vee \boldsymbol{\beta}_{k}=\gamma}}\left(\left|w_{\beta_{1}} \| w_{\beta_{2}}\right|\right)^{2^{p-2}}\right)^{1 / 2^{p-2}} \prod_{k=1}^{p-2}\|w\|_{2^{k}} \\
& \leq\|w\|_{2^{p-1}} \prod_{k=1}^{p-1}\|w\|_{2^{k}} .
\end{aligned}
$$


Therefore,

$$
\begin{aligned}
\|z w\|_{p}^{p} & \leq \sum_{\alpha_{1}, \ldots, \alpha_{p} \in \mathfrak{I}_{0}}\left|z_{\alpha_{1}}\right| \cdots\left|z_{\alpha_{p}}\right|\|w\|_{2^{p-1}} \prod_{k=1}^{p-1}\|w\|_{2^{k}} \\
& \leq\left(\sum_{\alpha \in \mathfrak{I}_{0}}\left|z_{\alpha}\right|\right)^{p}\|w\|_{2^{p-1}} \prod_{k=1}^{p-1}\|w\|_{2^{k}} \\
& \leq\|z\|_{1}^{p}\|w\|_{2^{p-1}} \prod_{k=1}^{p-1}\|w\|_{2^{k}},
\end{aligned}
$$

proving (2.7). The proof of (2.8) follows in a similar manner.

Remark 2.17. The inequalities presented in Theorem 2.16 are homogeneous.

Remark 2.18. The inequalities (2.7) and (2.8) are not enough to state that, in the closure $\bar{\Lambda}^{(p)}$ of $\Lambda$ with respect to the $p$ norm, the product of elements is a law of composition, i.e., if $z, w \in \bar{\Lambda}^{(p)}$, the aforementioned inequalities do not guarantee that the product converges in $\bar{\Lambda}^{(p)}$. In this paper, we are particularly interested in $\bar{\Lambda}^{(2)}$. However, we were unable to show whether the product is or is not a law of composition in this space. Although the solution to this question is important and can reveal interesting aspects of $\bar{\Lambda}^{(2)}$, the main results we present are independent of it.

Remark 2.19. In Ref. 24, a Fréchet structure modeled on a therein defined sequence space is given to $\Lambda$ by endowing it with seminorms.

Up to now, besides the last remarks, we only considered algebraic properties of $\Lambda$. To study convergence problems, it is necessary to complete this set. The completion with respect to the 1-norm, i.e., the space $\bar{\Lambda}^{(1)}$, has a Banach algebra structure and is already known in the literature. It was introduced by Rogers. ${ }^{34,38}$ In Sec. III, we study $\bar{\Lambda}^{(2)}$.

\section{THE FOCK SPACE}

The classical Fock space associated with $\ell_{2}$, i.e., the reproducing kernel Hilbert space introduced by Bargmann with the reproducing kernel ${ }^{11}$

$$
e^{\langle z, w\rangle \ell_{2}}=\sum_{\alpha \in \ell} \frac{z^{\alpha} \bar{w}^{\alpha}}{\alpha !},
$$

corresponds to function theory in a (countably) infinite number of commuting complex variables. In (3.1), we have used the notation of several complex variables and set $z=\left(z_{1}, z_{2}, \ldots\right) \in \ell_{2}(\mathbb{N}), \ell$ to be the family of sequences

$$
\alpha=\left(\alpha_{1}, \alpha_{2}, \ldots\right), \quad \alpha_{j} \in \mathbb{N}_{0}
$$

where at most a finite number of $\alpha_{j}$ are different from 0 ,

$$
z^{\alpha}=z_{1}^{\alpha_{1}} z_{2}^{\alpha_{2}} \cdots
$$

and $\alpha !=\alpha_{1} ! \alpha_{2} ! \cdots$.

In the noncommutative setting, the commuting variables give place to the noncommuting ones and, then, one needs a different set of indexes $\widetilde{\ell}$. We consider $\alpha \in \widetilde{\ell}$ given by

$$
\alpha=\left(\left(\alpha_{1}, n_{1}\right),\left(\alpha_{2}, n_{2}\right), \ldots,\left(\alpha_{m}, n_{m}\right)\right)
$$

where $n_{m} \in \mathbb{N}$ and $\alpha_{u} \neq \alpha_{u+1}$, for $u=1, \ldots, m-1$. Hence, the new kernel is written as 


$$
\sum_{\alpha \in \widetilde{\ell}} z^{\alpha} \overline{w^{\alpha}}
$$

where here $\overline{w^{\alpha}}=\cdots{\overline{w_{2}}}^{n_{2}}{\overline{w_{1}}}^{n_{1}}$.

Note that the left concatenation gives $\tilde{\ell}$ a monoid structure. Furthermore, it defines a partial order as follows: For $\alpha, \beta \in \tilde{\ell}$, we say that $\beta \leq \alpha$ if there is $\gamma \in \widetilde{\ell}$ such that $\alpha=\beta \gamma$.

The parallel with the structure of the Grassmann algebra is clear. Motivated by it, we give an inner product to $\bar{\Lambda}^{(2)}$ envisioning the construction of the counterpart of the Fock space here.

Definition 3.1. The inner product $\langle\cdot, \cdot\rangle$ between two supernumbers $z, w \in \Lambda$ is defined as

$$
\langle z, w\rangle=\sum_{\alpha \in \mathfrak{I}_{0}} z_{\alpha} \overline{w_{\alpha}} .
$$

Remark 3.2. Let $z$ and $w$ be two supernumbers. Then,

$$
\langle w, z\rangle=\left\langle z^{\dagger_{2}}, w^{\dagger^{2}}\right\rangle=\left\langle z^{\dagger_{4}}, w^{\dagger_{4}}\right\rangle=\left\langle z^{\dagger_{5}}, w^{\dagger_{5}}\right\rangle=\left\langle z^{\dagger_{7}}, w^{\dagger_{7}}\right\rangle .
$$

Proposition 3.3. $\bar{\Lambda}^{(2)}$ endowed with the aforementioned defined inner product and the 2-norm is a Hilbert space. For simplicity of notation, we denote such a Hilbert space simply by $\bar{\Lambda}^{(2)}$.

Proof. It suffices to observe that the 2-norm is induced by the above defined inner product. In fact,

$$
\|z\|_{2} \equiv\langle z, z\rangle^{1 / 2} .
$$

Definition 3.4. By analogy with the noncommutative setting, as discussed in the beginning of this section (and also noting the definition in Ref. 44$), \bar{\Lambda}^{(2)}$ is called the Fock space.

In general, we consider functions $f: \mathcal{I} \rightarrow \bar{\Lambda}^{(2)}$, where $\mathcal{I}$ is the domain of $f$, usually $\mathbb{C}$ or $\mathbb{R}$. Whenever we write $f \in \bar{\Lambda}^{(2)}$, we mean that $f(x) \in \bar{\Lambda}^{(2)}$ for every $x \in \mathcal{I}$.

As in the classical examples of Fock space, it is possible to define the left multiplication operation $M_{f}$ in $\bar{\Lambda}^{(2)}$. But there is a caveat: as already discussed, the multiplication might not be a law of composition in $\bar{\Lambda}^{(2)}$. If that is the case, $M_{f}$ is unbounded for an arbitrary $f \in \bar{\Lambda}^{(2)}$. One can, then, use two different approaches: study such operators in a space of stochastic distributions $\mathfrak{S}_{-1}$, as we discuss in Sec. IV, or restrict $\mathrm{M}_{f}$ to $f \in \bar{\Lambda}^{(1)} \subset \bar{\Lambda}^{(2)}$ since Theorem 2.16 assures that $\mathrm{M}_{f}$ is bounded in this case, i.e., if $f \in \bar{\Lambda}^{(1)} \subset \bar{\Lambda}^{(2)}$ and $g \in \bar{\Lambda}^{(2)}$, we have

$$
\mathrm{M}_{f} g=f g \in \bar{\Lambda}^{(2)} .
$$

In this section, we consider only $\mathrm{M}_{f}$ with $f \in \bar{\Lambda}^{(1)}$. When we get back to it in Sec. V, we consider the general case where $f \in \bar{\Lambda}^{(2)}$ since the product is a law of composition in the space of stochastic distributions, which is introduced in Sec. IV.

Note that, if $f=\sum_{\alpha \in \mathfrak{I}_{0}} f_{\alpha} i_{\alpha}$,

$$
\mathrm{M}_{f}=\sum_{\alpha \in \mathfrak{I}_{0}} f_{\alpha} \mathrm{M}_{i_{\alpha}} .
$$

Therefore, we can focus our analysis on multiplication by elements of $\mathfrak{I}_{0}$. Furthermore, we note that the focus can be on the set $\mathfrak{I}$, since multiplication by the generator of the body, i.e., $M_{1}$ is just the identity operator.

In the case of elements associated with $\mathfrak{I}_{0}$, we already know that

$$
i_{\alpha} i_{\beta}=(-1)^{\sigma(\alpha, \beta)} \sum_{\eta \in \mathfrak{I}} \delta_{\alpha \vee \beta, \eta} i_{\eta} .
$$


It is also straightforward from the definition of the inner product that

$$
\left\langle i_{\alpha}, i_{\beta}\right\rangle=\delta_{\alpha, \beta}
$$

Then,

$$
\left\langle\mathrm{M}_{i_{\alpha}} i_{\beta}, i_{\gamma}\right\rangle=(-1)^{\sigma(\alpha, \beta)} \sum_{\eta \in \mathfrak{I}} \delta_{\alpha \vee \beta, \eta}\left\langle i_{\eta}, i_{\gamma}\right\rangle=(-1)^{\sigma(\alpha, \beta)} \delta_{\alpha \vee \beta, \gamma} .
$$

We can also look for an expression for the adjoint $M_{i_{\alpha}}^{*}$ of $M_{i_{\alpha}}$

$$
\left\langle\mathrm{M}_{i_{\alpha}}^{*} i_{\beta}, i_{\gamma}\right\rangle=\left\langle i_{\beta}, \mathrm{M}_{i_{\alpha}} i_{\gamma}\right\rangle=(-1)^{\sigma(\alpha, \gamma)} \delta_{\beta, \alpha \vee \gamma} .
$$

By analogy with the classical cases, we say $M_{i_{\alpha}}^{*}$ is the left derivative with respect to $i_{\alpha}$.

Observing that

$$
M_{i_{\alpha}}=M_{i_{a_{1}}} M_{i_{a_{2}}} \ldots M_{i_{a_{\mathrm{t}}}}
$$

and, as a consequence,

$$
M_{i_{\alpha}}^{*}=M_{i_{a_{t}}}^{*} M_{i_{a_{t-1}}}^{*} \ldots M_{i_{a_{1}}}^{*}
$$

we can pay close attention to the left derivative with respect to single generators. Then,

$$
\mathrm{M}_{i_{n}}^{*} i_{\alpha}=\left\{\begin{array}{ll}
0, & \text { if } a_{k} \neq n, \forall a_{k} \in\{1, \ldots,|\alpha|\} \\
(-1)^{k-1} i_{a_{1}} i_{a_{2}} \cdots i_{a_{k-1}} i_{a_{k+1}} \ldots i_{a_{|\alpha|}}, & \text { if } \exists k ; a_{k}=n
\end{array} .\right.
$$

Therefore, the left derivative constructed here corresponds to the one that is traditionally defined in superanalysis and its applications. $^{12,13,16,38}$

Remark 3.5. Expression (3.7) clearly shows that the operator conjugation $*$ is an extension of the involution of supernumbers $\dagger_{7}$.

Remark 3.6. The Berezin integral is a concept widely used in superanalysis and supersymmetry. ${ }^{12,13,16,38}$ It coincides with the left derivative and, then, can also be defined in terms of $\mathrm{M}_{i_{n}}^{*}$

$$
\int d i_{n} f \equiv M_{i_{n}} f
$$

More generally, if $|\alpha|<\infty$,

$$
\int d i_{\alpha} f \equiv \int d i_{a_{|\alpha|}} \ldots \int i_{a_{1}} f=M_{i_{a_{|\alpha|} \mid}^{*}}^{*} \cdots M_{i_{a_{1}}}^{*} f
$$

Moreover, if $f$ is generated by $i_{a_{1}}, \ldots, i_{a_{N}}$ and $i_{\alpha}=i_{a_{1}} \ldots i_{a_{N}}$, the Berezin integral

$$
\int d i_{\alpha} f=f_{1,2, \ldots, N}
$$

reduces to

$$
\int d i_{\alpha} f=\left\langle M_{f} 1, i_{\alpha}\right\rangle
$$

which has some resemblance to a residue.

It is also worth considering the self-adjoint operator

$$
\mathrm{T}_{f}=\mathrm{M}_{f}+\mathrm{M}_{f}^{*}
$$


Proposition 3.7. Let $f, g \in \bar{\Lambda}^{(2)}$ with $f_{\mathrm{B}}=g_{\mathrm{B}}=0$, then the following equation holds:

$$
\left\langle\mathrm{T}_{f} 1, \mathrm{~T}_{g} 1\right\rangle=\langle f, g\rangle .
$$

Proof. First, note that $M_{f}^{*} 1=f_{B}$ and $M_{g}^{*} 1=g_{B}$. Then, if $f_{B}=g_{B}=0$,

$$
\left\langle\mathrm{T}_{f} 1, \mathrm{~T}_{g} 1\right\rangle=\left\langle f+f_{\mathrm{B}}, g+g_{\mathrm{B}}\right\rangle=\langle f, g\rangle .
$$

Such an operator $\mathrm{T}_{f}$ is used in Sec. V when we define stochastic processes associated with the Fock space.

\section{A TOPOLOGICAL ALGEBRA ASSOCIATED WITH $\wedge$}

Let $\mathscr{S}$ denote the space of Schwartz functions (the space of test functions), and let $\mathscr{S}$ be its dual (the space of tempered distributions). The Gel'fand triple $\left(\mathscr{S}, \mathbf{L}_{2}(\mathbb{R}, d x), \mathscr{S}\right)$ plays an important role in classical analysis, see, for instance, Ref. 39. Gel'fand triples are also defined in Hida's white noise space theory ${ }^{23,28}$ and in its noncommutative counterpart, 5,8 and are used to solve stochastic differential equations and model stochastic processes and their derivatives. In this section, we define Gel'fand triples in the Grassmann setting, with the aim of solving similar problems. Most of the results presented here are parallel to others discussed in Refs. 9 and 10.

One of the reasons commonly used for introducing a Gel'fand triple is the fact that in a Hilbert space, one can define products different from the inner product between two elements and, often, those products are not a law of composition on such a space, i.e., the result of the product of two elements does not necessarily belong to the Hilbert space. For instance, in the white noise space, the Wick product is not a law of composition. Then, one embeds the white noise space into a space of stochastic distributions to make it a law of composition. Various choices are possible to do so.

In our case, as already discussed, it is not yet clear if the product is a law of composition in $\bar{\Lambda}^{(2)}$. So one could question if it is necessary to make use of an analogous embedding. However, making the product a law of composition is not the only reason to introduce the space of stochastic distributions. In fact, when considering stochastic processes, such spaces are necessary for the study of their derivatives. Because of it, we introduce in this section the analogous of the space of stochastic distributions. Before doing so, we review a few facts from the classical case as well as from the theory of perfect spaces and strong algebras. We refer the reader to Refs. 18 and 19 for more information on these spaces.

Our starting point is a decreasing family of Hilbert spaces $\left(\mathcal{H}_{p},\|\cdot\|_{\mathcal{H}_{p}}\right)_{p \in \mathbb{Z}}$, with increasing norms. The intersection $\mathcal{F}=$ $\cap_{p=0}^{\infty} \mathcal{H}_{p}$ is a Fréchet space, which we assume perfect, meaning that compactness is equivalent to being bounded and compact. This will happen, in particular, when for every $p$, there exists $q>p$ such that the injection map from $\mathcal{H}_{q}$ into $\mathcal{H}_{p}$ is compact. An important instance is when the injection is nuclear. We identify $\mathcal{H}_{p}^{\prime}$ with $\mathcal{H}_{-p}$. Our main interest is the dual $\mathcal{F}^{\prime}=\cup_{p=0}^{\infty} \mathcal{H}_{-p}$, which together with $\mathcal{F}$ and $\mathcal{H}_{0}$, for a Gel'fand triple.

We endow the dual $\mathcal{F}^{\prime}$ with the strong topology, defined in terms of the bounded sets of $\mathcal{F}$. The space $\mathcal{F}^{\prime}$ is then locally convex, and the strong topology coincides with the inductive limit topology. See Ref. 9, Sec. III, for a discussion.

Therefore, the analysis is, a priori, done in a larger space of distributions, which is a (non-metrizable) inductive limit of Hilbert spaces (distributions here being understood as continuous functionals on the space $\mathcal{F}^{\prime}$ ). However, it is in fact done locally in a Hilbert space. There are two reasons why this happens. The first reason is that the space of distributions is the dual of a Fréchet nuclear space (dual of a perfect space would suffice). The second reason is the algebra structure of $\mathfrak{S}_{-1}$, and Våge inequality (4.3). We now state two of the main results related to such spaces. They are used in the Proofs of Theorems 5.6 and 5.5.

Proposition 4.1. A set is (weakly or strongly) compact in $\mathcal{F}^{\prime}$ if and only if it is compact in one of the spaces $\mathcal{H}_{-p}$ in the corresponding norm.

Proposition 4.2. Assume $\mathcal{F}^{\prime}$ perfect. Then, weak and strong convergence of sequences is equivalent, and a sequence converges (weakly or strongly) if and only if it converges in one of the spaces $\mathcal{H}_{-p}$ in the corresponding norm.

A topological algebra is assumed to be separately continuous in each variable. It is not a trivial fact that a strong algebra is in fact jointly continuous in the two variables [see Ref. 14, IV.26, Theorem 2, and the discussion in Ref. 10, pp. 215-216]. 
In the case we are interested, we define

$$
\mathcal{H}_{-p}\left(\mathrm{c}_{\alpha}\right)=\left\{f=\left.\sum_{\alpha \in \mathfrak{I}_{0}} f_{\alpha} i_{\alpha} \in \Lambda^{(2)}\left|\sum_{\alpha \in \mathfrak{I}_{0}}\right| f_{\alpha}\right|^{2} c_{\alpha}^{-2 p}<\infty\right\},
$$

where $p \in \mathbb{Z}$ and the coefficients $\mathrm{c}_{\alpha}$ 's form a sequence of positive real numbers such that

$$
\mathrm{c}_{\alpha} \mathrm{c}_{\beta} \leq \mathrm{c}_{\gamma}
$$

if $\alpha \vee \beta=\gamma$ and

where $d$ is a positive integer. Observe that

$$
\sum_{\alpha \in \mathfrak{I}_{0}} c_{\alpha}^{-2 d}<\infty
$$

if $p \geq q$.

$$
\mathcal{H}_{-q}\left(c_{\alpha}\right) \subseteq \mathcal{H}_{-p}\left(c_{\alpha}\right)
$$

Henceforth, we denote $\mathcal{H}_{-p}\left(c_{\alpha}\right)$ simply by $\mathcal{H}_{-p}$.

Definition 4.3. The norm $\|f\|_{\mathcal{H}_{-p}}$ of $f \in \mathcal{H}_{-p}$ is defined as

$$
\|f\|_{\mathcal{H}_{-p}} \equiv \sum_{\alpha \in \mathfrak{I}_{0}}\left|f_{\alpha}\right|^{2} c_{\alpha}^{-2 p} .
$$

Proposition 4.4. If $c_{\alpha \vee \beta}=c_{\alpha} c_{\beta}$, then $c_{0}=1$.

Proof. On the one hand, if $c_{0}>1$, the inequality (4.1) does not hold in general since

$$
\mathrm{c}_{0} \mathrm{c}_{\alpha}>\mathrm{c}_{\alpha}=\mathrm{c}_{0 \vee \alpha} .
$$

On the other hand, if $c_{0}<1$, the condition (4.2) is not satisfied. In fact, $c_{0}^{-2 d}<c_{\alpha}^{-2 d}$ for every $\alpha \in \mathfrak{I}$ and then, the sum in (4.2) diverges.

Proposition 4.5. Let $f \in \mathcal{H}_{-p}$ with $c_{\alpha}>1$ if $\alpha \neq 0$ and $c_{0}=1$. Then,

$$
\lim _{p \rightarrow \infty}\|f\|_{\mathcal{H}_{-p}}=\left|f_{0}\right|^{2} .
$$

Proof. Because $\lim _{p \rightarrow \infty} c_{\alpha}^{-2 p}=0$ for every $\alpha \neq 0$,

$$
\begin{aligned}
\lim _{p \rightarrow \infty}\|f\|_{\mathcal{H}_{-p}} & =\lim _{p \rightarrow \infty} \sum_{\alpha \in \mathfrak{I}_{0}}\left|f_{\alpha}\right|^{2} c_{\alpha}^{-2 p} \\
& =\sum_{\alpha \in \mathfrak{I}_{0}}\left|f_{\alpha}\right|^{2} \lim _{p \rightarrow \infty} c_{\alpha}^{-2 p} \\
& =\left|f_{0}\right|^{2} .
\end{aligned}
$$

Theorem 4.6 introduces a Våge-like inequality, which is the analogous of a result due to Våge ${ }^{43}$ and allows the analysis of stochastic processes to be done locally in a Hilbert space.

Theorem 4.6. If $f \in \mathcal{H}_{-q}$ and $g \in \mathcal{H}_{-p}$, with $p>q$, then

$$
\|f g\|_{\mathcal{H}_{-p}} \leq \mathrm{C}_{p-q}\|f\|_{\mathcal{H}_{-q}}\|g\|_{\mathcal{H}_{-p}},
$$

where $\mathrm{C}_{p-q}$ is a positive constant. 
Proof. Let $f \in \mathcal{H}_{-q}$ and $g \in \mathcal{H}_{-p}$. Hence, using the Cauchy-Schwarz inequality,

$$
\begin{aligned}
& \|f g\|_{\mathcal{H}_{-p}}^{2}=\sum_{\gamma \in \mathfrak{I}_{0}}\left|(f g)_{\gamma}\right|^{2} c_{\gamma}^{-2 p} \\
& =\sum_{\gamma \in \mathfrak{I}_{0}}\left|\sum_{\alpha \vee \beta=\gamma}(-1)^{\sigma(\alpha, \beta)} f_{\alpha} g_{\beta}\right|^{2} c_{\gamma}^{-2 p} \\
& \leq \sum_{\gamma \in \mathfrak{I}_{0}}\left(\sum_{\substack{\alpha \vee \beta=\gamma \\
\alpha^{\prime} \vee \beta^{\prime}=\gamma}}\left|f_{\alpha} \| g_{\beta}\right|\left|f_{\alpha^{\prime}}\right|\left|g_{\beta^{\prime}}\right|\right) c_{\gamma}^{-2 p} \\
& \leq \sum_{\gamma \in \mathfrak{I}_{0}}\left(\sum_{\substack{\alpha \vee \beta=\gamma \\
\alpha^{\prime} \vee \beta^{\prime}=\gamma}}\left|f_{\alpha}\right| c_{\alpha}^{-p}\left|g_{\beta}\right| c_{\beta}^{-p}\left|f_{\alpha^{\prime}}\right| c_{\alpha^{\prime}}^{-p}\left|g_{\beta^{\prime}}\right| c_{\beta^{\prime}}^{-p}\right) \\
& \leq \sum_{\alpha, \alpha^{\prime} \in \mathfrak{I}_{0}}\left|f_{\alpha}\right| c_{\alpha}^{-p}\left|f_{\alpha^{\prime}}\right| \mathrm{c}_{\alpha^{\prime}}^{-p}\left(\sum_{\substack{\gamma \in \mathfrak{I}_{0} ; \exists \beta, \beta^{\prime} \\
\alpha \vee \beta=\gamma \\
\alpha^{\prime} \vee \beta^{\prime}=\gamma}}\left|g_{\beta}\right| \mathrm{c}_{\beta}^{-p}\left|g_{\beta^{\prime}}\right| \mathrm{c}_{\beta^{\prime}}^{-p}\right) \\
& \leq \sum_{\alpha, \alpha^{\prime} \in \mathfrak{J}_{0}}\left|f_{\alpha}\right| c_{\alpha}^{-p}\left|f_{\alpha^{\prime}}\right| c_{\alpha^{\prime}}^{-p}\left(\sum_{\substack{\gamma \in \mathcal{I}_{0} ; \exists \beta \\
\alpha \vee \beta=\gamma}}\left|g_{\beta}\right|^{2} c_{\beta}^{-2 p}\right)^{1 / 2}\left(\sum_{\substack{\gamma \in \mathcal{I}_{0} ; \exists \exists \beta^{\prime} \\
\alpha^{\prime} \vee \beta^{\prime}=\gamma}}\left|g_{\beta^{\prime}}\right|^{2} c_{\beta^{\prime}}^{-2 p}\right)^{1 / 2} \\
& \leq \sum_{\alpha, \alpha^{\prime} \in \mathfrak{I}_{0}}\left|f_{\alpha}\right| \mathrm{c}_{\alpha}^{-p}\left|f_{\alpha^{\prime}}\right| c_{\alpha^{\prime}}^{-p}\left(\sum_{\beta \in \mathfrak{I}_{0}}\left|g_{\beta}\right|^{2} c_{\beta}^{-2 p}\right)^{1 / 2}\left(\sum_{\beta^{\prime} \in \mathfrak{I}_{0}}\left|g_{\beta^{\prime}}\right|^{2} c_{\beta^{\prime}}^{-2 p}\right)^{1 / 2} \\
& \leq\left(\sum_{\alpha \in \mathfrak{I}_{0}}\left|f_{\alpha}\right| c_{\alpha}^{-p}\right)^{2}\|g\|_{\mathcal{H}_{-p}}^{2} \\
& \leq\left(\sum_{\alpha \in \mathcal{I}_{0}}\left|f_{\alpha}\right| c_{\alpha}^{-q} c_{\alpha}^{q-p}\right)^{2}\|g\|_{\mathcal{H}_{-p}}^{2} \\
& \leq\left(\sum_{\alpha \in \mathfrak{I}_{0}} c_{\alpha}^{-2(p-q)}\right)\|f\|_{\mathcal{H}_{-q}}^{2}\|g\|_{\mathcal{H}_{-p}}^{2} .
\end{aligned}
$$

Now, it remains to be shown that there exist $c_{\alpha}$ 's such that $\sum_{\alpha \in \mathfrak{I}_{0}} c_{\alpha}^{-2(p-q)}<\infty$. We recall that $\alpha \in \mathfrak{I}$ means that $\alpha=\left(a_{1}, \ldots, a_{n}\right)$ for some integer $n \geq 1$, where $a_{1}, \ldots, a_{n}$ are positive integers such that $a_{1}<a_{2}<\cdots<a_{n}$. The "extension" $\mathfrak{I}_{0}$ adds one more element to I, namely, $\alpha=0$. With this in mind, one possible family of weights $\mathrm{c}_{\alpha}$ that satisfy (4.1) and (4.2) is given by

$$
\mathrm{c}_{\alpha}=e^{\sum_{k=1}^{n} \varphi\left(a_{k}\right)}
$$

for every $\alpha \in \mathfrak{I}_{0}$, where $\varphi$ is a monotonically increasing real power series-or at least, the values $\varphi(n)$, for non-negative integers $n$, form a monotonically increasing sequence-with $\varphi(0)=0$. We also require the coefficients of the power series to be bigger than $\ln 2^{1 / 2(p-q)}$.

Then, it is straightforward that

$$
\mathrm{c}_{\alpha} \mathrm{c}_{\beta}=\mathrm{c}_{\gamma}
$$

if $\alpha \vee \beta=\gamma$ and $c_{0}=1$, as required by Proposition 4.4. Moreover, if $d=p-q>0$,

$$
\sum_{\alpha \in \mathfrak{I}_{0}} c_{\alpha}^{2(q-p)}=1+\sum_{n=1}^{\infty} \sum_{\substack{\alpha \in \mathfrak{I} ; \\|\alpha|=n}} e^{-2 d \sum_{k=1}^{n} \varphi\left(a_{k}\right) .}
$$

Note that there exists $\xi>\ln 2^{1 / 2 d}$ such that 


$$
\sum_{\substack{\alpha \in \mathfrak{I}_{;} \\|\alpha|=1}} e^{-2 d \varphi\left(a_{1}\right)}=\sum_{a_{1}=1}^{\infty} e^{-2 d \varphi\left(a_{1}\right)} \leq \sum_{a_{1}=1}^{\infty} e^{-2 d \xi a_{1}}=\frac{1}{e^{2 d \xi}-1}<1 .
$$

Also,

and, in general,

$$
\sum_{\substack{\alpha \in \mathcal{J} ; \\|\alpha|=2}} e^{-2 d\left(\varphi\left(a_{1}\right)+\varphi\left(a_{2}\right)\right)} \leq\left(\sum_{a_{1}=1}^{\infty} e^{-2 d \varphi\left(a_{1}\right)}\right)\left(\sum_{a_{2}=1}^{\infty} e^{-2 d \varphi\left(a_{2}\right)}\right) \leq\left(\frac{1}{e^{2 d \xi}-1}\right)^{2}
$$

$$
\sum_{\substack{\alpha \in \mathfrak{T} ; \\|\alpha|=n}} e^{-2 d\left(\varphi\left(a_{1}\right)+\cdots+\varphi\left(a_{n}\right)\right)} \leq\left(\sum_{a_{1}=1}^{\infty} e^{-2 d \varphi\left(a_{1}\right)}\right) \cdots\left(\sum_{a_{n}=1}^{\infty} e^{-2 d \varphi\left(a_{n}\right)}\right) \leq\left(\frac{1}{e^{2 d \xi}-1}\right)^{n} .
$$

$$
\sum_{\alpha \in \mathfrak{I}_{0}} c_{\alpha}^{-2 d} \leq 1+\sum_{n=1}^{\infty}\left(\frac{1}{e^{2 d \xi}-1}\right)^{n}=1+\frac{1}{e^{2 d \xi}-2}
$$

We present Corollary 4.7, whose proof is analogous to the one just presented for Theorem 4.6.

Corollary 4.7. If $f \in \mathcal{H}_{-p}$ and $g \in \mathcal{H}_{-q}$, with $p>q$, then

$$
\|f g\|_{\mathcal{H}_{-p}} \leq C_{p-q}\|f\|_{\mathcal{H}_{-p}}\|g\|_{\mathcal{H}_{-q}},
$$

where $\mathrm{C}_{p-q}$ is a positive constant.

Definition 4.8. We define the space

and its topological dual

$$
\mathfrak{S}_{1}=\cap_{p \in \mathbb{Z}} \mathcal{H}_{p}
$$

$$
\mathfrak{S}_{-1}=\cup_{p \in \mathbb{Z}}, \mathcal{H}_{-p}
$$

which are, respectively, the analogues of the space of test functions and the space of tempered distributions in the classical cases.

Corollary 4.9. The space $\mathfrak{S}_{-1}$ endowed with the product is a strong algebra.

Outline of the proof. We first endow $\mathfrak{S}_{-1}$ with the inductive topology. Theorem 4.6 implies that the product is separately continuous in each $\mathcal{H}_{-p}$, which is equivalent to continuity in the inductive topology. Furthermore, $\mathfrak{S}_{-1}$ inherits the associativity of the product in $\Lambda$. We have, then, a Banach algebra structure. Thus, $\mathfrak{S}_{-1}$ can be seen as the inductive limit of Banach spaces, which makes it a strong algebra. See Ref. 10 for more details.

Remark 4.10. Note that the inductive topology is equivalent to the strong topology.

Remark 4.11. The product would also be associative in $\bar{\Lambda}^{(2)}$ if it was a law of composition there.

Remark 4.12. If one considers the strong convolution algebra associated with $\Im_{0}$ endowed with the convolution $\vee$, one obtains a strong algebra that is closely related to $\mathfrak{S}_{-1}$. Although those two algebras are not isomorphic as a ring, they are isomorphic as locally convex topological vector spaces. As a consequence, it follows from Ref. 9, Theorem 3.7, that $\mathfrak{S}_{-1}$ is nuclear and, hence, perfect.

Corollary 4.13. Let $n \in \mathbb{N}$ and $f \in \mathcal{H}_{-p} \subseteq \mathcal{H}_{-p-2}$. Then,

$$
\left\|f^{n}\right\|_{\mathcal{H}_{-p-2}} \leq C_{2}^{n-1}\|f\|_{\mathcal{H}_{-p}}^{n} .
$$

Proof. First, note that $\|f\|_{\mathcal{H}_{-p-2}} \leq\|f\|_{\mathcal{H}_{-p}}$ for every $f \in \mathcal{H}_{-p} \subseteq \mathcal{H}_{-p-2}$. Hence, using Theorem 4.6, 


$$
\begin{aligned}
\left\|f^{n}\right\|_{\mathcal{H}_{-p-2}} & \leq C_{2}\|f\|_{\mathcal{H}_{-p}}\left\|f^{n-1}\right\|_{\mathcal{H}_{-p-2}} \\
& \leq C_{2}^{2}\|f\|_{\mathcal{H}_{-p}}^{2}\left\|f^{n-2}\right\|_{\mathcal{H}_{-p-2}} \\
& \leq C_{2}^{n-1}\|f\|_{\mathcal{H}_{-p}}^{n} .
\end{aligned}
$$

Corollary 4.14. Let

$$
\mathrm{F}(\lambda)=\sum_{n \in \mathbb{N}_{0}} \alpha_{n} \lambda^{n}
$$

be an absolutely convergent power series in the open disk with radius $R$, where $\alpha_{n}, \lambda \in \mathbb{C}$ and $\mathbb{N}_{0}=\mathbb{N} \cup\{0\}$. If $f \in \mathcal{H}_{-p}$, then $F(f)$ converges in $\mathcal{H}_{-p-2}$ if

$$
\|f\|_{\mathcal{H}_{-p}}<\frac{R}{C_{2}} .
$$

Proof. By assumption, if $|\lambda|<R$, the power series (4.5) converges absolutely, i.e.,

$$
\sum_{n \in \mathbb{N}_{0}}\left|\alpha_{n} \lambda^{n}\right|=\sum_{n \in \mathbb{N}_{0}}\left|\alpha_{n}\right|\left|\lambda^{n}\right|<\infty .
$$

Using Corollary 4.13, we can study the absolute convergence of $\mathrm{F}(f)$ in $\mathcal{H}_{-p}$ :

$$
\begin{aligned}
\sum_{n \in \mathbb{N}_{0}}\left\|\alpha_{n} f^{n}\right\|_{\mathcal{H}_{-p-2}} & =\sum_{n \in \mathbb{N}_{0}}\left|\alpha_{n}\right|^{2}\left\|f^{n}\right\|_{\mathcal{H}_{-p-2}} \\
& \leq \alpha_{0}+C_{2}^{-1} \sum_{n \in \mathbb{N}}\left|\alpha_{n}\right|^{2}\left(C_{2}\|f\|_{\mathcal{H}_{-p}}\right)^{n} .
\end{aligned}
$$

Then, $F(f)$ converges absolutely in $\mathcal{H}_{-p-2}$ if

$$
\mathrm{C}_{2}\|f\|_{\mathcal{H}_{-p}}<\mathrm{R} \Rightarrow\|f\|_{\mathcal{H}_{-p}}<\frac{\mathrm{R}}{\mathrm{C}_{2}} .
$$

Corollary 4.15. Let $F(\lambda)$ be a power series as in Corollary 4.14. Then, $F(f)$ converges in $\mathfrak{S}_{-1}$ for $f \in \mathfrak{S}_{-1}$ if the body of $f$ satisfies (4.6).

Proof. If $f \in \mathfrak{S}_{-1}$, there exists an integer $q_{0}$ such that $f \in \mathcal{H}_{-q}$ for every $q \geq q_{0}$. By Theorem 4.14, for $\mathrm{F}(f)$ to converge, it is necessary that $\|f\|_{\mathcal{H}_{-q}}<R / C_{2}$, which does not hold in general. However, because of Proposition 4.5 , we can reduce this condition to

proving the corollary.

$$
\left|f_{0}\right|^{2}<\frac{R}{C_{2}}
$$

Corollary 4.16. Let $f \in \mathfrak{S}_{-1}$. Then, $f$ is invertible if and only if its body $f_{0}$ satisfies $f_{0} \neq 0$.

Proof. On the one hand, if $g$ is the inverse of $f$ and its body is given by $g_{0}$, then

$$
f g=1 \Rightarrow f_{0} g_{0}=1 \Rightarrow f_{0} \neq 0 .
$$

On the other hand, if $f_{0} \neq 0$, consider without loss of generality $f_{0}=1$. Then, Corollary 4.15 implies that

$$
F(f)=\sum_{n \in \mathbb{N}_{0}}(1-f)^{n}
$$

converges if the body of $1-f$ is smaller than $\mathrm{C}_{2}^{-1}$. However, $(1-f)_{B}=0$. Therefore, $g=F(f) \in \mathfrak{S}_{-1}$ and $g$ is the inverse of $f$. 


\section{STOCHASTIC PROCESSES AND THEIR DERIVATIVES}

In the literature and, in particular, the work of Rogers (see, e.g., Refs. 35-37), a class of $\Lambda$-valued functions of a real variable is considered and defined as stochastic processes. Here, we look at $\bar{\Lambda}^{(2)}$-valued functions and present a different approach to stochastic processes in the Grassmannian setting. Our aim is to obtain a close counterpart of the noncommutative white noise space theory. 5,8

Let us start reviewing this framework-we refer the reader to Refs. 5 and 44 for more details. First, we recall that the study of Gaussian stochastic processes can be made through the analysis of positive-definite kernels since there is a oneto-one correspondence between the two notions. ${ }^{29,33}$ In fact, the kernel coincides with the covariance of the stochastic process.

In the framework we are basing our model, as can be seen in Ref. 1, Sec. III, the processes are associated with positive-definite kernels of the form

$$
K_{\sigma}(t, s)=\int_{\mathbb{R}} \frac{\left(e^{i u t}-1\right)\left(e^{-i u s}-1\right)}{u^{2}} d \sigma(u),
$$

where $\sigma$ is absolutely increasing continuous with respect to the Lebesgue measure, $d \sigma(u)=m(u) d u$, such that the Stieltjes integral

$$
\int_{\mathbb{R}} \frac{m(u) d u}{u^{2}+1}<\infty .
$$

The reason for such choice is the fact that integrals of the form (5.1) correspond to correlation functions of zero-mean Gaussian processes with stationary increments (see Refs. 27 and 32). An important example of such processes is the fractional Brownian motion, for which $d \sigma(u)=|u|^{1-2 H} d u$, with $H \in(1,2)$. In this case, the correlation function $K_{\sigma}(t, s)$ becomes

$$
K(t, s)=\gamma_{H}\left(|t|^{2 H}+|s|^{2 H}-|t-s|^{2 H}\right),
$$

where $\gamma_{H}$ depends only on $H$ and, if $H \neq 1 / 2$, is equal to

$$
\gamma_{\mathrm{H}}=\frac{\cos (\pi \mathrm{H}) \Gamma(2-2 \mathrm{H})}{(1-2 \mathrm{H}) \mathrm{H}}
$$

where $\Gamma$ is the Euler's gamma function. Moreover, $\gamma_{1 / 2}=\pi$ by continuity.

We, then, define an operator $S_{m}$ in $\mathbf{L}_{2}(\mathbb{R})$ such that

$$
\widehat{S_{m} f}(u)=\sqrt{m(u) \hat{f}}(u),
$$

where $\widehat{f}$ is the Fourier transform of $f$. Note that $S_{m}$ is, in general, unbounded. Its domain is

$$
\operatorname{dom} S_{m}=\left\{\left.f \in \mathbf{L}_{2}(\mathbb{R})\left|\int_{\mathbb{R}} m(u)\right| \widehat{f}(u)\right|^{2} d u<\infty\right\},
$$

which contains $\mathbf{1}_{[0, t]}$. Defining

$$
f_{m}(t)=S_{m} \mathbf{1}_{[0, t]}
$$

and using Plancherel's equality,

$$
\begin{aligned}
& \left\langle f_{m}(t), f_{m}(s)\right\rangle_{\mathbf{L}_{2}(\mathbb{R})}=\frac{1}{2 \pi}\left\langle\widehat{f}_{m}(t), \widehat{f}_{m}(s)\right\rangle_{\mathbf{L}_{2}(\mathbb{R})} \\
& =\frac{1}{2 \pi}\left\langle\sqrt{m(u)} \widehat{\mathbf{1}}_{[0, t]}, \sqrt{m(u)} \widehat{\mathbf{1}}_{[0, s]}\right\rangle_{\mathbf{L}_{2}(\mathbb{R})} \\
& =\frac{1}{2 \pi}\left\langle m(u) \frac{e^{-i u t}-1}{u}, \frac{e^{-i u s}-1}{u}\right\rangle_{\mathbf{L}_{2}(\mathbb{R})} \\
& =\frac{1}{2 \pi} \int_{\mathbb{R}} \frac{\left(e^{i u t}-1\right)\left(e^{-i u s}-1\right)}{u^{2}} m(u) d u .
\end{aligned}
$$


In order to obtain the stochastic processes we are interested in, we construct a random variable associated with the functions $f_{m}(t)$. This is done with the introduction of the creation operator $\ell_{h}$, with $h \in \mathbf{L}_{2}(\mathbb{R})$, defined by

$$
\ell_{h}(f)=h \otimes f, \quad f \in \Gamma\left(\mathbf{L}_{2}(\mathbb{R})\right)
$$

where $\Gamma\left(\mathbf{L}_{2}(\mathbb{R})\right)$ denotes the full Fock space associated with $\mathbf{L}_{2}(\mathbb{R})$. Finally, letting $T_{h}=\ell_{h}+\ell_{h}^{*}$, we define a random variable $\mathrm{X}_{m}(\mathrm{t})$ as

$$
\mathrm{X}_{m}(\mathrm{t}) \equiv \mathrm{T}_{f_{m}(t)}
$$

Observe that the expected value of a random variable $X_{m}(t)$ can be defined by

$$
\mathrm{E}\left(\mathrm{X}_{m}(\mathrm{t})\right)=\left\langle\Omega, \mathrm{T}_{f_{m}(\mathrm{t})}(\Omega)\right\rangle_{\Gamma}
$$

where $\Omega$ is the vacuum state of $\Gamma$. Moreover, as expected

$$
\mathrm{E}\left(\mathrm{X}_{m}(\mathrm{t}) \mathrm{X}_{m}(\mathrm{~s})\right)=\left\langle\mathrm{T}_{f_{m}(\mathrm{t})}(\Omega), \mathrm{T}_{f_{m}(s)}(\Omega)\right\rangle_{\Gamma}=\left\langle f_{m}(\mathrm{t}), f_{m}(\mathrm{~s})\right\rangle_{\mathbf{L}_{2}(\mathbb{R})}=\mathrm{K}_{\sigma}(\mathrm{t}, \mathrm{s}),
$$

where $K_{\sigma}(t, s)$ is given by (5.1) with $d \sigma(u)=m(u) d u$, as already discussed.

The stochastic free processes associated with those variables have the concept of freeness-in opposition to independenceassociated with them. Moreover, instead of Gaussian distributions, they have semi-circle distributions. Again, we refer the reader to Refs. 3 and 44 for an extended discussion on this topic.

In the case of the stochastic processes we desire to define in the setting of the Grassmann numbers, we replace the operator $\mathrm{T}_{f}$ in expression (5.5) by the operator we defined in expression (3.8) with

$$
\begin{aligned}
f_{m}(t) & =\sum_{n \in \mathbb{N}}\left\langle S_{m} \mathbf{1}_{[0, t]}, \xi_{n}\right\rangle_{\mathbf{L}_{2}(\mathbb{R})} i_{n} \\
& =\sum_{n \in \mathbb{N}}\left\langle\mathbf{1}_{[0, t]}, S_{m} \xi_{n}\right\rangle_{\mathbf{L}_{2}(\mathbb{R})} i_{n} \\
& =\sum_{n \in \mathbb{N}}\left(\int_{0}^{t}\left(S_{m} \xi_{n}\right)(u) d u\right) i_{n},
\end{aligned}
$$

where $\xi_{n}$ denotes the Hermite functions.

Hence,

$$
\mathrm{X}_{m}(\mathrm{t})=\sum_{n \in \mathbb{N}}\left(\int_{0}^{t}\left(\mathrm{~S}_{m} \xi_{n}\right)(u) d u\right) \mathrm{T}_{i_{n}} .
$$

We can also define the expected value function $E$ in a similar manner as defined in the "classical" case

$$
\mathrm{E}\left(\mathrm{X}_{m}(\mathrm{t})\right)=\left\langle 1, \mathrm{X}_{m}(\mathrm{t}) 1\right\rangle_{\bar{\Lambda}^{(2)}}
$$

where 1 is the vacuum state in the Fock space introduced in Sec. III. Note that $E\left(X_{m}(t)\right)=0$.

Using (3.7), we observe that the covariance, which gives the kernel $K(t, s)$, satisfies

$$
\mathrm{E}\left(\mathrm{X}_{m}(\mathrm{t}) \mathrm{X}_{m}(\mathrm{~s})\right)=\mathrm{K}(\mathrm{t}, \mathrm{s})=\left\langle\mathrm{X}_{m}(\mathrm{t}) 1, \mathrm{X}_{m}(\mathrm{~s}) \boldsymbol{1}_{\bar{\Lambda}^{(2)}}=\left\langle f_{m}(\mathrm{t}), f_{m}(\mathrm{~s})\right\rangle_{\bar{\Lambda}^{(2)}}\right.
$$

Hence,

$$
K(t, s)=\sum_{n \in \mathbb{N}}\left(\int_{0}^{t}\left(S_{m} \xi_{n}\right)(u) d u\right)\left(\int_{0}^{s}\left(S_{m} \xi_{n}\right)\left(u^{\prime}\right) d u^{\prime}\right)
$$

which is equivalent to the kernel (5.1).

Now, we gather a few results concerning bounds for the operator $\mathrm{T}_{f}$, where $f \in \mathcal{H}_{-p}$ for some $p \in \mathbb{N}$. They are relevant in the Proof of Theorem 5.5.

Proposition 5.1. For every $f \in \mathfrak{S}_{-1}$ with $\|f\|_{\mathcal{H}_{-p}}<\infty$, the operator $\mathrm{M}_{f}$ is bounded from $\mathcal{H}_{p}$ into $\mathcal{H}_{-p}$. 
Proof. Let $g \in \mathcal{H}_{p}$. Then, using Corollary 4.7, we have

$$
\left\|\mathrm{M}_{f} g\right\|_{\mathcal{H}_{-p}}=\|f g\|_{\mathcal{H}_{-p}} \leq \mathrm{C}_{2 p}\|f\|_{\mathcal{H}_{-p}}\|g\|_{\mathcal{H}_{p}} .
$$

For the next result, and henceforth, we consider spaces $\mathcal{H}_{-p}$ where the coefficients $c_{\alpha}$ of the norm are of the type introduced in the Proof of Theorem 4.6. In particular, if $\alpha \vee \beta=\gamma, c_{\alpha} c_{\beta}=c_{\gamma}$.

Proposition 5.2. For every $f \in \mathcal{H}_{-q}$, the operator $\mathrm{M}_{f}^{*}$ is bounded from $\mathcal{H}_{p}$ into $\mathcal{H}_{-p}$, where $q<p$.

Proof. Let $g \in \mathcal{H}_{p}$. Then, we have

$$
\begin{aligned}
\left\|M_{f}^{*} g\right\|_{\mathcal{H}_{-p}}^{2} \leq\left\|M_{f}^{*} g\right\|_{\mathcal{H}_{p}}^{2} & =\sum_{\gamma \in \mathfrak{I}_{0}}\left|\sum_{\alpha \vee \gamma=\beta}(-1)^{\sigma(\alpha, \gamma)} f_{\alpha} g_{\beta}\right|^{2} c_{\gamma}^{2 p} \\
& \leq \sum_{\gamma \in \mathfrak{I}_{0}}\left(\sum_{\alpha \vee \gamma=\beta}\left|f_{\alpha}\right|\left|g_{\beta}\right|\right)^{2} c_{\gamma}^{2 p} \\
& \leq \sum_{\alpha, \alpha^{\prime} \in \mathfrak{I}_{0}}\left|f_{\alpha}\right| c_{\alpha}^{-p}\left|f_{\alpha}^{\prime}\right| c_{\alpha^{\prime}}^{-p} \sum_{\substack{\gamma \in \mathfrak{I}_{0} ; \exists \beta, \beta^{\prime} \\
\alpha \vee \gamma=\beta \\
\alpha^{\prime} \gamma \gamma=\beta^{\prime}}}\left|g_{\beta}\right| c_{\beta}^{p}\left|g_{\beta}^{\prime}\right| c_{\beta^{\prime}}^{p} \\
& \leq \sum_{\alpha, \alpha^{\prime} \in \mathcal{I}_{0}}\left|f_{\alpha}\right| c_{\alpha}^{-p}\left|f_{\alpha}^{\prime}\right| c_{\alpha^{\prime}}^{-p}\|g\|_{\mathcal{H}_{p}}^{2} \\
& \leq\left(\sum_{\alpha \in \mathfrak{I}_{0}}\left|f_{\alpha}\right| c_{\alpha}^{-q} c_{\alpha}^{-(p-q)}\right)^{2}\|g\|_{\mathcal{H}_{p}}^{2} \\
& \leq C_{p-q}^{2}\|f\|_{\mathcal{H}_{-q}}^{2}\|g\|_{\mathcal{H}_{p} .}^{2} .
\end{aligned}
$$

Corollary 5.3. For every $f \in \mathfrak{S}_{-1}$, the operator $T_{f}=M_{f}+M_{f}^{*}$ is bounded from $\mathfrak{S}_{1}$ into $\mathfrak{S}_{-1}$ and there exists $p>q$ such that

$$
\left\|\mathrm{T}_{f} g\right\|_{-p} \leq 2 \mathrm{C}_{1}\|f\|_{-q}\|g\|_{p} .
$$

Proof. This is a direct consequence of Proposition 5.1 and Proposition 5.2. However, since Proposition 5.1 is, in some sense, stronger than what we need, we note that if $f \in \mathcal{H}_{-q}$ and $g_{\in} \mathcal{H}_{p}$, the following equation holds from Proposition 5.2 and Theorem 4.6:

$$
\begin{aligned}
\left\|\mathrm{T}_{f} g\right\|_{-p} & \leq\left\|\mathrm{M}_{f} g\right\|_{-p}+\left\|\mathrm{M}_{f}^{*} g\right\|_{-p} \\
& \leq \mathrm{C}_{p-q}\|f\|_{-q}\|g\|_{-p}+C_{p-q}\|f\|_{-q}\|g\|_{p} \\
& \leq 2 C_{p-q}\|f\|_{-q}\|g\|_{p} \\
& \leq 2 C_{1}\|f\|_{-q}\|g\|_{p} .
\end{aligned}
$$

To compute $d \mathrm{X}_{m}(t) / d t$, we will make use of the spaces $\mathfrak{S}_{1}$ and $\mathfrak{S}_{-1}$ and assume certain growth conditions for $m$. First, we present Proposition 5.4, the proof for which we refer the reader to Ref. 2 (Proposition 3.7 and Lemma 3.8):

Proposition 5.4. Let $m$ satisfy

$$
m(u) \leq \begin{cases}K|u|^{-b} & |u| \leq 1, \\ K|u|^{2 \mathrm{~N}} & |u|>1,\end{cases}
$$

where $b<2, \mathrm{~N} \in \mathbb{N}_{0}$, and $\mathrm{K}$ is a positive real constant. Then,

$$
\left|\mathrm{S}_{m} \xi_{n}(t)\right| \leq \mathrm{D}_{1} n^{\frac{\mathrm{N}+1}{2}}+\mathrm{D}_{2}
$$

and 


$$
\left|\mathrm{S}_{m} \xi_{n}(\mathrm{t})-\mathrm{S}_{m} \xi_{n}(\mathrm{~s})\right| \leq|t-\mathrm{s}|\left(\mathrm{D}_{3} n^{\frac{\mathrm{N}+2}{2}}+\mathrm{D}_{4}\right),
$$

where $D_{1}, D_{2}, D_{3}$, and $D_{4}$ are non-negative functions independent of $n$.

Theorem 5.5. Let $m$ be a positive measurable function, satisfying (5.7) and (5.2) [the latter for $d \sigma(t)=m(t) d t$ ]. Then, for every $g \in \mathfrak{S}_{1}$, the function $t \mapsto X_{m}(t) f$ is strongly continuous in $\mathfrak{S}_{-1}$ and there exists a continuous operator $W_{m}(t)$ from $\mathfrak{S}_{1}$ into $\mathfrak{S}_{-1}$ such that

$$
\frac{d}{d t} \mathrm{X}_{m}(\mathrm{t}) g=\mathrm{W}_{m}(\mathrm{t}) g .
$$

Finally, the function $t \mapsto \mathrm{W}_{m}(\mathrm{t}) \mathrm{g}$ is continuous from $[a, b]$ into $\mathfrak{S}_{-1}$.

Proof. Since $f_{m}(t)=\sum_{n \in \mathbb{N}} f_{n}^{m}(t) i_{n}$, with $f_{n}^{m}=\int_{0}^{t} S_{m} \xi_{n}(u) d u$, we use (5.8) to obtain

$$
\left\|\frac{d}{d t} f_{m}(t)\right\|_{\mathcal{H}_{-p}}^{2}=\sum_{n \in \mathbb{N}}\left|S_{m} \xi_{n}(t)\right|^{2} c_{n}^{-2 p} \leq \sum_{n \in \mathbb{N}}\left(D_{1} n^{\frac{N+1}{2}}+D_{2}\right)^{2} c_{n}^{-2 p}
$$

Then, for every suitable choice of coefficients $c_{n}$, there exists a positive integer $p_{0}$ such that, for every $p \geq p_{0}, d f_{m}(t) / d t \in \mathcal{H}_{-p}$. Moreover, for $s=t+h$, where $h \neq 0$ is a real number, there exists $p_{1}$ such that

$$
K_{p}=\sum_{n \in \mathbb{N}}\left(D_{3} n^{\frac{N+2}{2}}+D_{4}\right)^{2} c_{n}^{-2 p}<\infty, \quad \forall p \geq p_{1}
$$

and, with (5.9), the $\mathcal{H}_{-p}$ norm of the difference of derivatives satisfies

$$
\sum_{n \in \mathbb{N}}\left|S_{m} \xi_{n}(s)-S_{m} \xi_{n}(t)\right|^{2} c_{n}^{-2 p} \leq K_{p_{1}}|h|^{2}
$$

Therefore, $\mathrm{W}_{m}=\mathrm{T}_{d f_{m} / d t}$ is a continuous operator from $\mathfrak{S}_{1}$ into $\mathfrak{S}_{-1}$.

Finally, to see that (5.10) holds, observe that for a real number $h \neq 0$ and $g \in \mathcal{H}_{p}$,

$$
\left(\frac{\mathrm{X}_{m}(\mathrm{t}+\mathrm{h})-\mathrm{X}_{m}(\mathrm{t})}{h}-\mathrm{W}_{m}(\mathrm{t})\right) g=\sum_{n \in \mathbb{N}} \frac{\int_{t}^{t+h}\left(\mathrm{~S}_{m} \xi(u)-\mathrm{S}_{m} \xi(\mathrm{t})\right) d u}{h} \mathrm{M}_{i_{n}} g=\mathrm{X}_{\Delta(t, h)} g,
$$

with

$$
\Delta(t, h)=\sum_{n \in \mathbb{N}} \frac{\int_{t}^{t+h}\left(S_{m} \xi(u)-S_{m} \xi(t)\right) d u}{h} i_{n} .
$$

Then, there exists $p>q \geq p_{1}$ such that, using Corollary 5.3,

$$
\left\|\mathrm{X}_{\Delta(t, h)} g\right\|_{\mathcal{H}_{-p}} \leq 2 C_{1}\|\Delta(t, h)\|_{\mathcal{H}_{-q}}\|g\|_{\mathcal{H}_{p}} \leq\left(2 C_{1} K_{p_{1}}\|g\|_{\mathcal{H}_{p}}\right)|h|^{2}
$$

Therefore, $\mathrm{W}_{m}(t)=\mathrm{T}_{d f_{m} / d t}(t) \equiv d \mathrm{X}_{m}(\mathrm{t}) / d t$ is a continuous operator from $\mathfrak{S}_{1}$ into $\mathfrak{S}_{-1}$. Finally, using Proposition 4.2 , one sees that the function $t \mapsto W_{m}(t) g$ is continuous.

Once the treatment for derivatives is formalized, the next natural step would be the development of the counterpart of the Ito/Malliavin stochastic calculus. The first step in this direction is the introduction of stochastic integrals. This is done in Theorem 5.6, while stochastic calculus itself will be developed elsewhere.

Theorem 5.6. Let $t \mapsto Y(t)$, with $t \in[a, b]$, be a continuous $\mathfrak{S}_{-1}$-valued function in the strong topology of $\mathfrak{S}_{-1}$. If $g \in \mathfrak{S}_{1}$, there exists a positive integer $p$, which depends on $g$, such that the Pettis integral

$$
\int_{a}^{b} \mathrm{Y}(\mathrm{t}) \mathrm{W}_{m}(\mathrm{t}) g d \mathrm{t}
$$

can be computed as a limit of Riemann sums and converges in $\mathcal{H}_{-p}$. 
Outline of the proof. As in Ref. 2, the function $t \mapsto W_{m}(t) g$ is continuous from $[a, b]$ to $\mathfrak{S}_{-1}$. Since the product is jointly continuous in $\mathfrak{S}_{-1}$, the map $t \mapsto \mathrm{Y}(\mathrm{t}) \mathrm{W}_{m}(\mathrm{t}) \mathrm{g}$ is continuous and its image is therefore compact in $\mathfrak{S}_{-1}$. By Proposition 4.1, there exists $p$ such that the image of $t \mapsto \mathrm{Y}(\mathrm{t}) \mathrm{W}_{m}(\mathrm{t}) \mathrm{g}$ is in $\mathcal{H}_{-p}$; this function is still continuous with respect to the topology of $\mathcal{H}_{-p}$, as is observed using Proposition 4.2. We then compute (5.11) in $\mathcal{H}_{-p}$ using Riemann sums.

As we already discussed, the random variables introduced here have the same expected value and the same covariance of the variables associated with free stochastic processes. Despite the fact that the product in the integral (5.11) is, ultimately, the product of $i_{\alpha}$ 's, what contrasts with the "classical" case, which is a product of Hermite functions, shows that the processes induced by them are very different.

\section{FINAL REMARKS}

In this paper, we have studied some aspects of the closure of the Grassmann algebra with respect to the 2-norm, which we called the Fock space, as well as its embedding in Gelfand triples. We also introduced a new type of stochastic process and an approach to study their derivatives. We present now research questions that emerge from the ideas discussed here.

An important matter that is still not resolved is whether the product is a law of composition in the Fock space. We know that even if there is no problem of convergence for the product, the evaluation of stochastic integrals could be problematic if we had not introduced the space of stochastic distributions. However, addressing this question is crucial and either answer will, surely, lead to new results on the structure of the Fock space introduced here.

Another direction that can be investigated is related to Wiener algebras. In Ref. 10, a special type of Wiener algebra which can be associated with any strong algebra was introduced in a generic framework. An open question is regarding whether the counterpart of such a Wiener algebra can reveal important aspects of the strong algebra we introduced, uncovering new aspects of it.

The stochastic processes we used as a basis have the concept of freeness-in opposition to independence-associated with their random variables, and the distribution associated with them are semi-circles-not Gaussians. A possible research direction is, then, the investigation of the following questions: What is the independence-like concept associated with the random variables defined here? What are the distributions associated with the stochastic processes generated by them? Furthermore, as already mentioned at the end of Sec. V, there is a possibility to develop the counterpart of the Ito and Malliavin stochastic calculus in the present setting. One could also look for the physical processes modeled by them.

Furthermore, another interesting direction is to look into generalizations of the processes defined here. We considered in Sec. $V$ the special cases where $d \sigma(t)=m(t) d t$. For a general $\sigma$, one cannot introduce the operator $S_{m}$ defined by (5.4). However, it is possible to prove that there exists a continuous positive operator A from the Schwartz space $\mathcal{S}$ into its dual $\mathcal{S}^{\prime}$ such that

$$
\left.\int_{\mathbb{R}} \widehat{f}(u)\right|^{2} d \sigma(u)=\langle\mathrm{Af}, f\rangle_{\mathcal{S}^{\prime}, \mathcal{S}}
$$

The operator A can be factorized via a Hilbert space since $\mathcal{S}$ is nuclear-see Ref. 20 for factorization theorems. An explicit construction of $\mathrm{A}$ in the form $\mathrm{A}=\mathrm{Q}_{\sigma}^{*} \mathrm{Q}_{\sigma}$, where $\mathrm{Q}_{\sigma}$ is continuous from $\mathcal{S}$ into $\mathbf{L}_{2}(\mathbb{R})$, is given in Ref. 4 .

Finally, a problem we intend to address soon in a different paper concerns the construction of the counterpart of linear systems and rational functions in the setting of the supernumbers. For the theory of linear systems in the setting of Hida's white noise theory and its noncommutative counterpart, see Refs. 26, 41, 42, and 6-8 for more recent studies.

\section{ACKNOWLEDGMENTS}

Daniel Alpay thanks the Foster G. and Mary McGaw Professorship in Mathematical Sciences, which supported this research. Ismael L. Paiva acknowledges financial support from the Science without Borders program (CNPq/Brazil). Daniele C. Struppa thanks the Donald Bren Distinguished Chair in Mathematics, which supported this research.

\section{REFERENCES}

${ }^{1}$ D. Alpay, H. Attia, and D. Levanony, "On the characteristics of a class of Gaussian processes within the white noise space setting," Stochastic Process. Appl. 120, 1074-1104 (2010).

${ }^{2}$ D. Alpay, H. Attia, and D. Levanony, "White noise based stochastic calculus associated with a class of Gaussian processes," Opuscula Math. 32(3), 401-422 (2012).

${ }^{3}$ D. Alpay, F. Colombo, and I. Sabadini, "On a class of quaternionic positive definite functions and their derivatives," J. Math. Phys. 58(3), 033501 (2017).

${ }^{4}$ D. Alpay, P. Jorgensen, and D. Levanony, “A class of Gaussian processes with fractional spectral measures," J. Funct. Anal. 261(2), 507-541 (2011).

${ }^{5}$ D. Alpay, P. Jorgensen, and G. Salomon, "On free stochastic processes and their derivatives," Stochastic Process. Appl. 124(10), 3392-3411 (2014). 
${ }^{6}$ D. Alpay and D. Levanony, "Linear stochastic systems: A white noise approach," Acta Appl. Math. 110(2), 545-572 (2010).

${ }^{7}$ D. Alpay, D. Levanony, and A. Pinhas, "Linear stochastic state space theory in the white noise space setting," SIAM J. Control Optim. 48, 5009-5027 (2010). ${ }^{8}$ D. Alpay and G. Salomon, "Non-commutative stochastic distributions and applications to linear systems theory," Stochastic Process. Appl. 123(6), 2303-2322 (2013).

${ }^{9}$ D. Alpay and G. Salomon, “Topological convolution algebras,” J. Funct. Anal. 264(9), 2224-2244 (2013).

${ }^{10}$ D. Alpay and G. Salomon, "On algebras which are inductive limits of Banach spaces," Integr. Equations Oper. Theory 83(2), 211-229 (2015).

${ }^{11}$ V. Bargmann, "Remarks on a Hilbert space of analytic functions," Proc. Natl. Acad. Sci. U. S. A. 48, 199-204 (1962).

${ }^{12}$ F. A. Berezin, The Method of Second Quantization (Academic Press, New York, 1966), p. 52.

${ }^{13}$ F. A. Berezin, Introduction to Superanalysis, Volume 9 of Mathematical Physics and Applied Mathematics (D. Reidel Publishing Co., Dordrecht, 1987). Edited and with a foreword by A. A. Kirillov, With an appendix by V. I. Ogievetsky, Translated from the Russian by J. Niederle and R. Kotecký, Translation edited by Dimitri Leǐtes.

${ }^{14} \mathrm{~N}$. Bourbaki, Espaces Vectoriels Topologiques (Masson, 1981).

${ }^{15} \mathrm{E}$. Cartan and J. Leray, La Théorie des Groupes Finis et Continus et la Géométrie Différentielle Traitées par la Méthode du Repère Mobile (Gauthier-Villars, 1937).

16B. DeWitt, Supermanifolds, Cambridge Monographs on Mathematical Physics, 2nd ed. (Cambridge University Press, Cambridge, 1992).

${ }^{17} \mathrm{~S}$. Gal, Introduction to Geometric Function Theory of Hypercomplex Variables (Nova Publishers, 2002), Vol. 10.

${ }^{18}$ I. M. Gel'fand and G. E. Shilov, Generalized Functions (Academic Press, 1968), Vol. 2.

${ }^{19}$ I. M. Gel'fand and N. Y. Vilenkin, Generalized Functions (Academic Press, New York, 1964), Vol. 4 [Applications of harmonic analysis (Harcourt Brace Jovanovich Publishers, 1977), Translated from the Russian by Amiel Feinstein].

${ }^{20} \mathrm{~J}$. Górniak and A. Weron, "Aronszajn-Kolmogorov type theorems for positive definite kernels in locally convex spaces," Stud. Math. 69(3), 235-246 (19801981).

${ }^{21}$ T. Hida, H. H. Kuo, and N. Obata, "Transformations for white noise functionals," J. Funct. Anal. 111(2), 259-277 (1993).

${ }^{22}$ T. Hida and Si. Si, Lectures on White Noise Functionals (World Scientific Publishing Co. Pte. Ltd., Hackensack, NJ, 2008).

${ }^{23} \mathrm{H}$. Holden, B. Øksendal, J. Ubøe, and T. Zhang, Stochastic Partial Differential equations, Probability and its Applications (Birkhäuser Boston, Inc., Boston, MA, 1996).

${ }^{24} \mathrm{~A}$. Inoue and Y. Maeda, "Foundations of calculus on super Euclidean space $\mathbf{R}^{m \mid n}$ based on a Fréchet-Grassmann algebra," Kodai Math. J. 14(1), 72-112 (1991).

${ }^{25} \mathrm{U}$. C. Ji and E. Lytvynov, "Wick calculus for noncommutative white noise corresponding to $q$-deformed commutation relations," Complex Anal. Oper. Theory 12, 1497-1517 (2016).

${ }^{26}$ R. E. Kalman, P. L. Falb, and M. A. Arbib, Topics in Mathematical System Theory (McGraw-Hill Book Co., New York, 1969).

${ }^{27}$ M. G. Krein, "On the problem of continuation of helical arcs in Hilbert space," C. R. (Dokl.) Acad. Sci. URSS 45, 139-142 (1944).

${ }^{28}$ H.-H. Kuo, White Noise Distribution Theory, Probability and Stochastics Series (CRC Press, Boca Raton, FL, 1996).

${ }^{29}$ M. Loève, Probability Theory. II, Graduate Texts in Mathematics, 4th ed. (Springer-Verlag, New York, 1978), Vol. 46.

30 J. L. Martin, "The Feynman principle for a Fermi system," Proc. R. Soc. London, Ser. A 251, 543-549 (1959).

${ }^{31}$ J. L. Martin, "Generalized classical dynamics, and the "classical analogue' of a Fermi oscillator," Proc. R. Soc. London, Ser. A 251, 536-542 (1959).

32. J. von Neumann and I. J. Schoenberg, "Fourier integrals and metric geometry," Trans. Am. Math. Soc. 50, 226-251 (1941).

33. Neveu, Processus Aléatoires Gaussiens (Les Presses de l'Université de Montréal, 1968).

${ }^{34}$ A. Rogers, "A global theory of supermanifolds," J. Math. Phys. 21(6), 1352-1365 (1980).

${ }^{35}$ A. Rogers, "Fermionic path integration and Grassmann Brownian motion," Commun. Math. Phys. 113(3), 353-368 (1987).

${ }^{36}$ A. Rogers, "Stochastic calculus and anticommuting variables," preprint arXiv:hep-th/9409162, 1994.

${ }^{37}$ A. Rogers, "Supersymmetry and brownian motion on supermanifolds," Infinite Dimens. Anal. Quantum Probab. Relat. Top. 6(supp01), 83-102 (2003).

${ }^{38}$ A. Rogers, Supermanifolds: Theory and Applications (World Scientific, Singapore, 2007).

${ }^{39} \mathrm{~L}$. Schwartz, Théorie des Distributions, Nouvelle édition, entiérement corrigée, refondue et augmentée (Publications de l'Institut de Mathématique de l'Université de Strasbourg, Hermann, Paris, 1966), No. IX-X.

${ }^{40} \mathrm{~J}$. Schwinger, "Particles and sources," Phys. Rev. 152(4), 1219 (1966).

${ }^{41}$ E. D. Sontag, "On linear systems and noncommutative rings," Math. Syst. Theory 9(4), 327-344 (1975/76).

${ }^{42}$ E. D. Sontag, "Linear systems over commutative rings: A survey," Ric. Autom. 7, 1-34 (1976).

${ }^{43}$ G. Våge, "Hilbert space methods applied to stochastic partial differential equations," in Stochastic Analysis and Related Topics, edited by H. Körezlioglu, B. Øksendal, and A. S. Üstünel (Birkäuser, Boston, 1996), pp. 281-294.

${ }^{44}$ D. V. Voiculescu, K. J. Dykema, and A. Nica. Free Random Variables, Volume 1 of CRM Monograph Series (American Mathematical Society, Providence, RI, 1992). A noncommutative probability approach to free products with applications to random matrices, operator algebras and harmonic analysis on free groups. 